\title{
Unique antineoplastic profile of Saquinavir-NO, a novel NO-derivative of the protease inhibitor Saquinavir, on the in vitro and in vivo tumor formation of A375 human melanoma cells
}

\author{
MARCO DONIA $^{1}$, KATIA MANGANO ${ }^{1}$, PAOLO FAGONE ${ }^{1}$, ROCCO DE PASQUALE ${ }^{2}$, \\ FRANCO DINOTTA $^{2}$, MARINELLA COCO ${ }^{1}$, JULIO PADRON ${ }^{3}$, YOUSEF AL-ABED ${ }^{4}$, \\ GIUSEPPE ANGELO GIOVANNI LOMBARDO ${ }^{5}$, DANIJELA MAKSIMOVIC-IVANIC ${ }^{6}$, \\ SANJA MIJATOVIC ${ }^{6}$, MAI-BRITT ZOCCA ${ }^{7}$, VINCENZO PERCIAVALLE ${ }^{1}$, \\ STANISLAVA STOSIC-GRUJICIC ${ }^{6}$ and FERDINANDO NICOLETTI ${ }^{1,7}$
}

\begin{abstract}
Departments of ${ }^{1}$ Bio-Medical Sciences, ${ }^{2}$ Dermatology Clinic, University of Catania, Catania; ${ }^{3}$ NicOx Research Institute, Bresso, Milan, Italy; ${ }^{4}$ Feinsten Institute for Medical Research, Laboratory of Medicinal Chemistry, North Shore Long Island Jewish Health System, Manhasset, NY, USA; ${ }^{5}$ Department of Plastic and Reconstructive Surgery, Cannizzaro Hospital, University of Catania, Catania, Italy; ${ }^{6}$ Department of Immunology, Institute for Biological Research 'Sinisa Stankovic', Belgrade University, Belgrade, Serbia; ${ }^{7}$ Onconox Aps, Copenhagen, Denmark
\end{abstract}

Received December 19, 2011; Accepted January 16, 2012

DOI: $10.3892 /$ or.2012.1840

\begin{abstract}
We have recently shown that covalent attachment of the nitric oxide (NO) moiety to the HIV protease inhibitor Saquinavir (Saq) produced a qualitatively new chemical entity, named Saquinavir-NO (Saq-NO), with enhanced anticancer properties and reduced toxicity both in vitro and in vivo. The aim of this study was to address several unanswered questions both on the pharmacological profile of Saq-NO as well as on the in vivo role of $\mathrm{NO}$ in the oncogenesis of A375 human melanoma cells. To this end, we have evaluated here the impact of single and combined effects of Saq-NO, Saq, the NO-donor DETA NONOate and the iNOS inhibitor L-NAME on the in vitro as well as in vivo growth of the iNOS positive A375 cells. Our data confirm clear-cut evidence for a strong and powerful anti-melanoma action of Saq-NO that is not duplicable by the combined use of Saq and DETA NONOate. Surprisingly, but also in agreement with the complex and multifaceted role of endogenous NO in A375 cells, both DETA NONOate and L-NAME significantly suppressed the in vivo growth of xenotransplants.
\end{abstract}

\section{Introduction}

The incidence of metastatic melanoma has increased dramatically over the past two decades (1), while the prognosis

Correspondence to: Professor Ferdinando Nicoletti, Department of Bio-Medical Sciences, University of Catania, Via Androne 83, 95124 Catania, Italy

E-mail: ferdinic@unict.it

Key words: nitric oxide, A375 cell line, melanoma, xenograft remained poor with an estimated 8600 persons died from melanoma in 2009 only in the United States (2). In 2010, the median survival of patients with melanoma who have distant metastases is still less than one year (3). Treatment with standard chemotherapeutic agents such dacarbazine, vinblastine or cisplatin yields low response rates (4). Cytokine therapy with high-dose IL-2 achieves similar response rates but it may induce, although with low frequency, durable complete response rates that lead to years of benefit (5). However, this treatment can be accompanied by severe toxicities that require the patient to be hospitalized for support during treatment (6). Cancer vaccines have also been largely studied with some promising results (7). Adoptive cell transfer (ACT) of tumor infiltrating lymphocytes in combination with IL-2 treatment after host preconditioning by lympho-depletion represents one of the most effective treatments for metastatic melanoma, but its application is limited by several disadvantages such as the high technical expertise required (8).

We have recently shown that covalent attachment of the nitric oxide (NO) moiety to the HIV protease inhibitor Saquinavir (Saq) produced a qualitatively new chemical entity (NCE), named Saquinavir-NO (Saq-NO) (Fig. 1), with enhanced anticancer properties and reduced toxicity. In particular, Saq-NO inhibited at significantly lower $\mathrm{IC}_{50}$ values compared to the parental compound the in vitro growth of rodent and human melanoma cell lines B16 and A375 $(9,10)$ showing potent anticancer effects also against multidrug-resistant cancer cells (11). Neither p53 mutation nor depletion nor expression of P-glycoprotein (P-gp), multidrug resistance-associated protein 1 (MRP1), or breast cancer resistance protein 1 (BRCP1) affected the anticancer activity of Saq-NO or Saq. Moreover, Saq-NO sensitized P-gp-, MRP1-, or BCRP1-expressing cancer cells to chemotherapy. Saq-NO enhanced sensitivity of P-gpor MRP1-expressing cancer cells to chemotherapy to a greater 
extent than Saq (11). In agreement with these in vitro effects, Saq-NO was more effective than its parental compound Saq in reducing the in vivo growth of the B16 and A375 xenotransplants $(9,10)$ as well as of the hormone resistant human prostate cancer PC3 cells both in vitro and in vivo (12).

In vitro studies on the B16 and A375 melanoma cell lines Saq-NO have shown a cell-dependent pharmacological profile of Saq-NO. While Saq-NO efficiently exerted Akt-independent cytostatic effects promoting terminal differentiation into Schwann-like cells in B16 cells (9), it exerted a strong cytotoxic effect on the inducible nitric oxide synthase (iNOS) positive A375 cells (10). In these cells, Saq-NO-triggered apoptosis was dependent on transient Akt up-regulation and reduced pERK and iNOS expression that were observed within the first 12 hours of exposure to the drug (10). Thereafter, however, Saq-NO up-regulated both iNOS transcription and NO endogenous synthesis and subsequently sensitized A375 cells to TRAIL (10). In all the settings considered, NO-release was modest thus qualifying Saq-NO as a soft NO-releaser drug.

The impact of Saq-NO on the in vitro and in vivo growth of A375 cells was of particular interest as these cells constitutively produce large amounts of NO that appears essential for exertion of its malignant potential (13). This is consistent with the emerging pathogenic role of endogenous NO in the development and clinical course of melanoma. In fact, constitutive expression of inducible form of iNOS correlates with poor survival (14-16). NO seems to initiate progression of human melanoma via a feedback loop mediated by apurinic/apyrimidinic endonuclease-1/ redox factor-1 (17).

Therefore, the evidence that a soft NO-releaser such as Saq-NO strongly influenced the in vitro and in vivo growth of the two melanoma cell lines B16 and A375 requires to be carefully weighed on other experimental studies before being further considered for translation to the clinical setting. This prompted us to carry out this phenomenological study where we evaluate the impact of single and combined effects of Saq-NO, Saq, the NO-donor DETA NONOate and the iNOS inhibitor L-NAME on the in vitro and in vivo growth of A375 cells xenotransplanted into nude mice.

The data confirm clear-cut evidence for a strong and powerful anti-melanoma action of Saq-NO and add important insights into its pharmacological profile. Surprisingly but fitting in with the complex and multifaceted role of endogenous NO in A375 cells, both DETA NONOate and L-NAME significantly suppressed the in vivo growth of these cells.

\section{Materials and methods}

Reagents and cells. Fetal calf serum (FCS), RPMI-1640, phosphate-buffered saline (PBS), were obtained from Sigma (Milan, Italy). Matrigel was obtained from BD Bioscience (San Jose, CA). Human melanoma A375 cell line was obtained from the American Type Culture Collection (Rockville, MD, USA). Cells were routinely maintained in RPMI-1640 medium supplemented with $10 \%$ FCS, 2 mM L-glutamine and antibiotics (culture medium) at $37^{\circ} \mathrm{C}$ in a humidified atmosphere with $5 \%$ $\mathrm{CO}_{2}$.

Test compounds. Nitro-L-arginine methyl ester (L-NAME) and DETA NONOate were obtained from Sigma. Saq was obtained

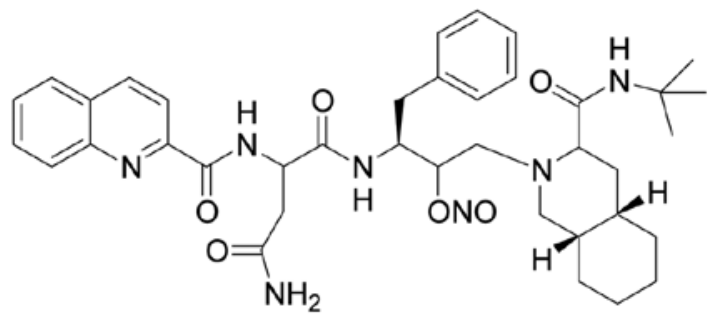

Saq-NO

$\mathrm{C}_{38} \mathrm{H}_{49} \mathrm{~N}_{7} \mathrm{O}_{7}$

Exact mass: 715.37

Figure 1. Structure of Saq-NO.
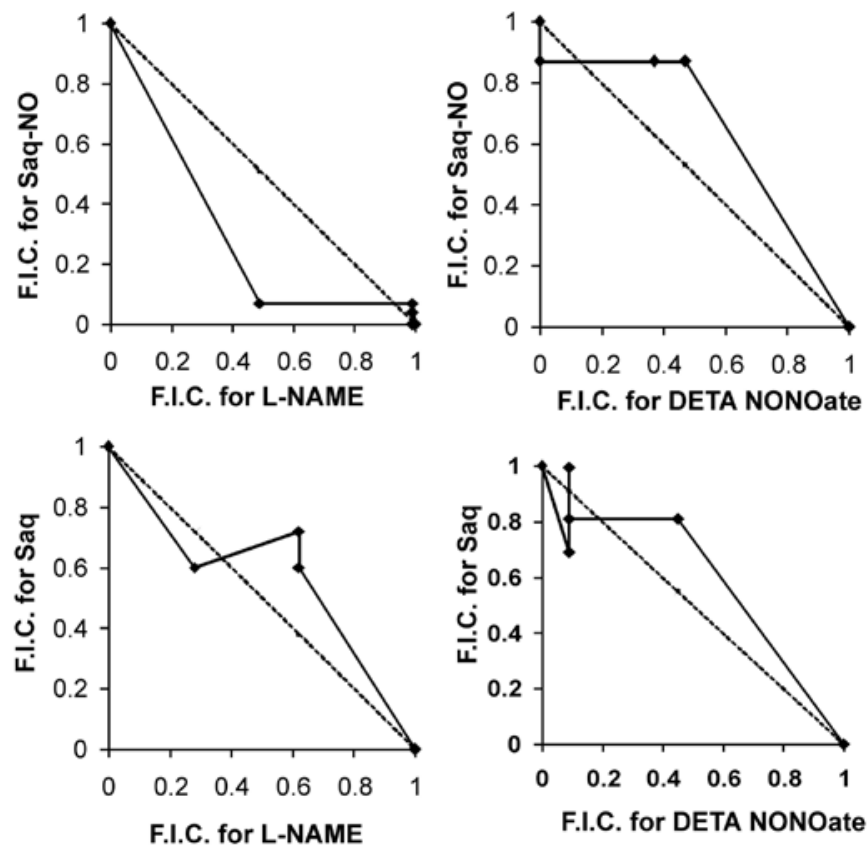

Figure 2. Antitumor activity of Saq and Saq-NO in the presence of L-NAME or DETA NONOate. Cells were treated with wide range of doses of experimental compounds for $24 \mathrm{~h}$ then cell viability was estimated by $\mathrm{CV}$ test and isobologram analysis was performed. F.I.C $<1$ is considered synergistic.

from Hoffmann La Roche. Saq-NO, synthesized as described elsewhere (9), was provided from Onconox Aps (Copenhagen, Denmark). Both Saq and Saq-NO were dissolved in DMSO $20 \%$. L-NAME and DETA NONOate were dissolved in water for injection.

In vitro studies and isobologram analysis. To classify the type of interaction between Saq-NO or Saq with L-NAME or DetaNONOate isobologram analysis was carried out as described elsewhere (10). Isobolograms were drawn from treatments with following concentration of Saq-NO $(4.7,9.4$, $18.8 \mu \mathrm{M})$ or the same concentrations of Saq with different concentrations of L-NAME $(5-20 \mu \mathrm{M})$ or DETA NONOate (125-500 $\mu \mathrm{M})$. Combinations reaching $30-50 \%$ of cytotoxicity were expressed as concentration of single agent alone provoking this toxicity. Analysis was carried out on the basis of doseresponse curves of cell viability treated with mentioned reagents 


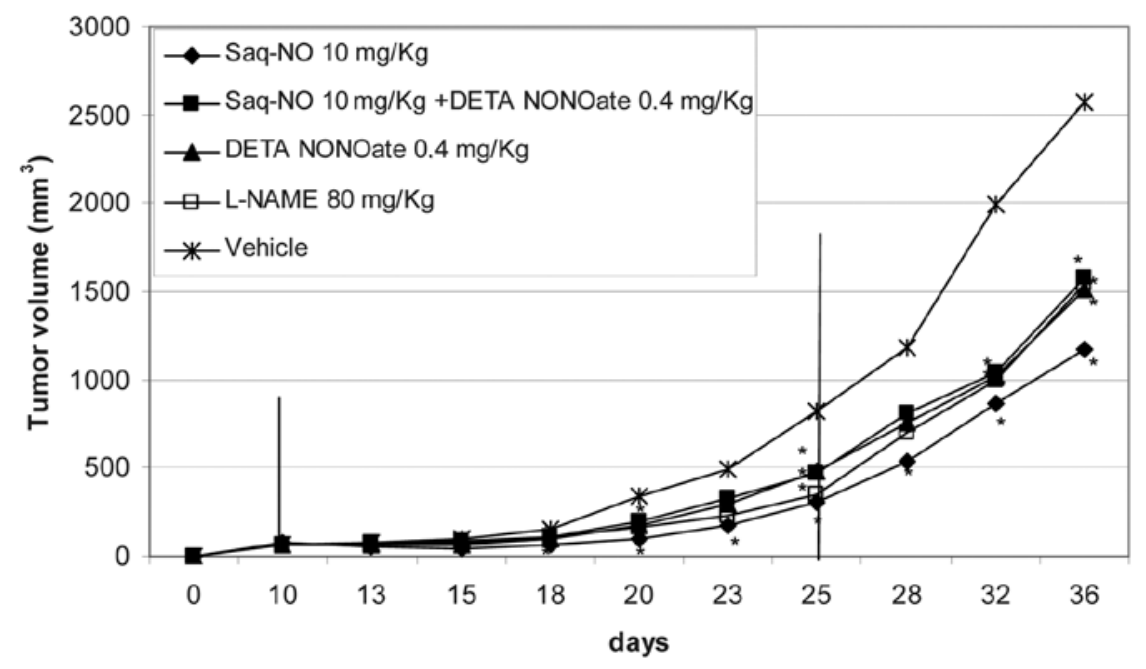

Figure 3. Saq-NO inhibited the growth of A375 melanoma cells in nude mice. Tumors were induced by subcutaneous implantation of $5 \times 10^{6}$ A375 melanoma cells, and the compounds were injected intraperitoneally for 16 consecutive days starting on day 8-10 following tumor implantation. Tumor volumes were measured twice a week for 34-36 days after tumor implantation. Each group consisted of 8-11 mice. * $\mathrm{p}<0.05$ by t-test refers to vehicle-treated animals.

for $24 \mathrm{~h}$. Cell viability was measured by crystal violet test as indicated previously (12).

Animals. Six- to eight-week-old male nude mice, weighing 25-28 g were purchased from Harlan-Nossan (San Pietro al Natisone, Udine, Italy). The mice were kept under standard laboratory conditions (non-specific pathogen-free) with free access to food and water. The animal studies were carried out in accordance to local guidelines and approved by local Institutional Animal Care and Use Committee.

Induction of melanoma and experimental treatment. On day 1 of the experiment, $5 \times 10^{6}$ A 375 melanoma cells were subcutaneously injected between the shoulder blades of each mouse using a $0.6-\mathrm{mm}$ needle. Cells were injected in a $200-\mu 1$ suspension consisting of $100 \mu \mathrm{l}$ of sterile Phosphate Buffered Saline (PBS) and $100 \mu \mathrm{l}$ of Matrigel, as previously described (18). Tumor growth was evaluated by measurement with calipers ( 2 perpendicular diameters) twice a week. Tumor volume was calculated using the formula $0.52 \mathrm{x} \mathrm{a} \mathrm{x} \mathrm{b}^{2}$, where $\mathrm{a}$ is the longest and $\mathrm{b}$ is the shortest diameter.

Several experimental groups of mice $(n=8$ to 11 mice for each group) were created that were treated by single and combined treatment with Saq, Saq-NO, DETA NONOate and L-NAME as described in Figs. 2 and 3. The control group was treated with DMSO 20\% that is the vehicle of Saq and Saq-NO. When the tumors were already palpable, 8 to 10 days after tumor induction, animals were randomly allocated to the different groups and were treated for 16 consecutive days. Post-randomization analysis revealed no significant differences in tumor volumes at the beginning of the treatment among the different groups. The study shown represents two independent experiments. Since the data were highly reproducible the results are merged and shown as a single experiment.

Statistical analysis. Students t-test was used to determine statistical significance. Values of $\mathrm{p}<0.05$ were considered to be statistically significant.

\section{Results}

Saq-NO down regulated A375 growth in vitro and in vivo. A375 cells were exposed to both Saq or Saq-NO in parallel with L-NAME or DETA NONOate. Cell viability was measured by crystal violet assay and isobologram curve was drawn. Saq-NO synergized with L-NAME while the effects with DETA NONOate was antagonistic (Fig. 2). Combined treatment of Saq with either L-NAME or DETA NONOate resulted in less toxicity then in monotreatment (Fig. 2).

To confirm the data obtained in vitro, we performed an in vivo xenograft model with cultured A 375 cells. In both in vivo studies with the A375 cells, tumor size in the vehicle-treated mice was $60-70 \mathrm{~mm}^{3}$ at the beginning of the treatment. Drugs were administered for a period of 16 consecutive days starting from 8 to 10 days after xenograft. Thereafter, the treatment was interrupted and the mice were followed up for additional 11 days to observe the growth of the tumor in the absence of the treatment. The readouts of the clinical results are summarized in Table I indicating the fold increase and Area Under the drug concentration-time Curve (AUC) of the tumors both at the end of the treatment period as well as at the end of the follow-up period. The statistical significance between the groups is summarized in Table II that indicates the days of observation during the treatment of significant inhibition of tumor growth. The kinetics of tumor development is shown in Figs. 3 and 4.

As seen from the observations of Table I and Figs. 3 and 4, Saq-NO appeared the most powerful of the treatments given either alone or in combination. This treatment was the most rapid of all those considered to achieve significant inhibition of tumor volume, with an effect prolonged for the entire period of treatment. This was also witnessed from the significant reduction of fold tumor increase (4 vs. 12) and AUC (1243 vs. 3671) observed at the end of the treatment as compared to vehicle-treated mice (Table I). A substantial increase of the tumor volumes was observed upon treatment withdrawal that led to a 19 -fold increase of the tumors in 
Table I. Summary of clinical readouts.

\begin{tabular}{lcccc}
\hline $\begin{array}{l}\text { Treatment } \\
\text { (16 days) }\end{array}$ & $\begin{array}{c}\text { Fold increase } \\
\text { (end of treatment) }\end{array}$ & $\begin{array}{c}\text { Fold increase } \\
\text { (end of study) }\end{array}$ & $\begin{array}{c}\text { AUC } \\
\text { (end of treatment) }\end{array}$ & $\begin{array}{c}\text { AUC } \\
\text { (end of study) }\end{array}$ \\
\hline Saq-NO & 4.4 & 19.0 & 1243 & 13759 \\
Saq-NO + DETA NONOate & 7.8 & 26.1 & 3358 & 24295 \\
Saq-NO + L-NAME & 3.5 & 12.3 & 733.3 & 8561 \\
Saq & 6.4 & 21.3 & 2551 & 17311 \\
Saq + DETA NONOate & 6.5 & 18.2 & 2340 & 16265 \\
Saq + L-NAME & 5.6 & 18.0 & 1974 & 14114 \\
DETA NONOate & 6.6 & 22.2 & 2283 & 18373 \\
L-NAME & 6.1 & 22.7 & 1778 & 16509 \\
Vehicle & 11.8 & 36.4 & 3671 & 26511 \\
\hline
\end{tabular}

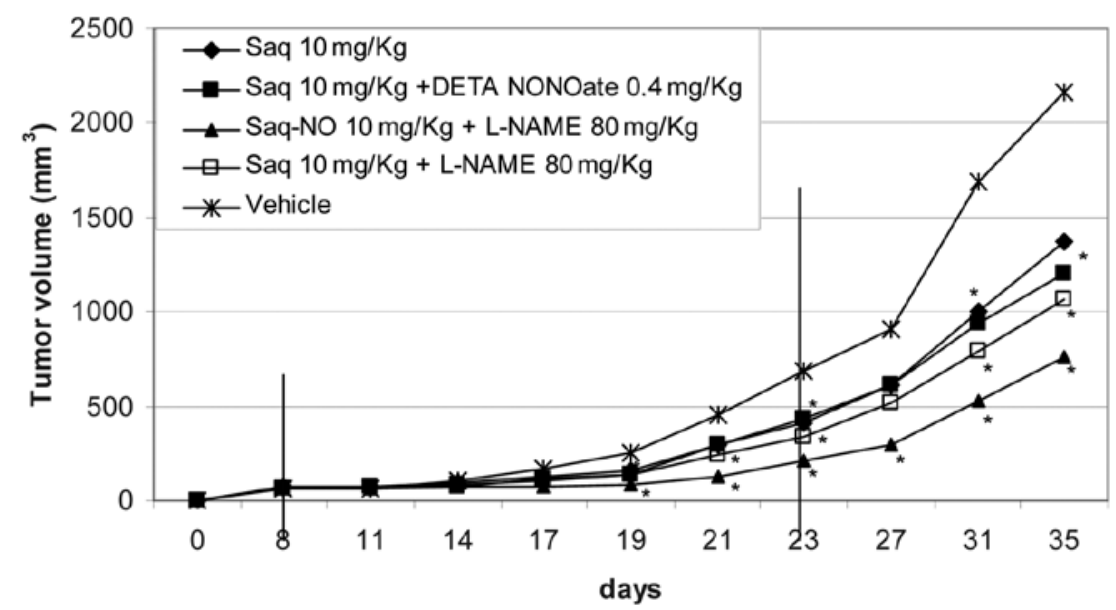

Figure 4. The association of Saq-NO and L-NAME inhibited the growth of A375 melanoma cells in nude mice. Tumors were induced by subcutaneous implantation of $5 \times 10^{6}$ A 375 melanoma cells, and the compounds were injected intraperitoneally for 16 consecutive days starting on day 8 -10 following tumor implantation. Tumor volumes were measured twice a week for 34-36 days after tumor implantation. Each group consisted of 8 - 11 mice. * $<<0.05$ by t-test refers to vehicle-treated animals.

the Saq-NO treated mice with an AUC of 13759. These values were however still significantly lower than those observed at the same period of time in the vehicle-treated mice that exhibited a 36-fold increase of the tumor volumes with an AUC of 26511 (Table I).

As we reported previously (10), the effects of Saq-NO were markedly stronger than those of its parental compound Saq. The mice treated with Saq exhibited a significant reduction of their tumor volumes on day 11 and 16 of the treatment. Although, relative to vehicle-treated mice, Saq favourably influenced the in vivo growth of A375 cells, its effects appeared of a significant lower magnitude than those of Saq-NO (Table II). Accordingly, both during the treatment period and at the end of the followup, the fold increase of the tumor volumes and the AUC were considerably higher than those observed in Saq-NO treated mice (see Table I and Figs. 3 and 4).

An additional group of mice was considered where the NO donor DETA NONOate was given alone or in combination to Saq or Saq-NO to mice xenotransplanted with A375 cells. On the one hand, these studies aimed at understanding whether the powerful anti-tumor effects of Saq-NO on the growth of A375 cells could have also been achieved by NO donation monotherapy, and on the other at evaluating whether the stronger in vivo anti-melanoma action of Saq-NO than Saq could be reproduced by the combined treatment of the latter with this prototypical NO donor. The data show that DETA NONOate monotherapy favourably influenced the growth of A375 cells at different time points during the study (days 11 and 16). The effects were however of considerable lower magnitude than those of Saq-NO and of comparable extent to those observed in mice treated with Saq (Table I and Fig. 3). The combined treatment with Saq and DETA NONOate failed to improve the chemotherapeutic efficacy of the drugs given alone (Table I and Fig. 4). Interestingly, and in agreement with the in vitro data, combined treatment with DETA NONOate reduced the anti-melanoma effects of Saq-NO (Table I and Fig. 3).

Finally, to evaluate the effects of NO inhibition in this experimental setting, we included groups of nude mice xenotransplanted with A375 cells treated with iNOS inhibitor L-NAME either alone or in combination with Saq or Saq-NO. In apparent contradiction with the beneficial effects of DETA NONOate, relative to treatment with vehicle, L-NAME 
Table II. Summary of statistical significance.

Inhibition of tumor growth during treatment (days)

\begin{tabular}{lccccc}
\cline { 2 - 5 } T-test vs. & Vehicle & Saq-NO & Saq & DETA NONOate & L-NAME \\
\hline Saq-NO & $6-16 \uparrow$ & - & $6,14 \uparrow$ & $16 \uparrow$ & $6 \uparrow$ \\
Saq-NO + DETA NONOate & NS & $6,14-16 \downarrow$ & - & NS & - \\
Saq-NO + L-NAME & $6-16 \uparrow$ & $9-11 \uparrow$ & - & - & $6-11 \uparrow$ \\
Saq & $11,16 \uparrow$ & $6,14 \downarrow$ & - & NS & NS \\
Saq + DETA NONOate & $11,16 \uparrow$ & - & NS & NS & - \\
Saq + L-NAME & $11,16 \uparrow$ & - & NS & - & NS \\
DETA NONOate & $11,16 \uparrow$ & $16 \downarrow$ & NS & - & NS \\
L-NAME & $11-16 \uparrow$ & $6 \downarrow$ & NS & NS & - \\
\hline
\end{tabular}

$\uparrow$, Higher tumor growth inhibition; $\downarrow$, Lower tumor growth inhibition.

monotherapy also significantly reduced at different time points during the study period the clinical readouts considered (Tables I and II; and Fig. 3). The effects were almost superimposable to those achieved with DETA NONOate treatment (Tables I and II; and Fig. 3). Interestingly, and extending to the in vivo setting the synergistic effect observed between Saq-NO and L-NAME in vitro, the combined treatment of L-NAME with Saq-NO (Tables I and II; and Fig. 4) appears to exert an additive effect so that AUC of the tumors of mice treated with Saq-NO + L-NAME was $\sim 70 \%$ lower than those observed with either of the two drugs in monotherapy (Table I and Fig. 4). However final reduction of tumor growth in combined treatment with Saq and L-NAME was not significantly potentiated in comparison to that found in monotherapy (Fig. 4).

In summary, in vitro evaluation of co-treatment of experimental drugs with NO donating compound or iNOS inhibitor showed a strong correlation and high reproducibility with results obtained in vivo.

\section{Discussion}

This study further characterizes the powerful antimelanoma action of Saq-NO, revealing important pharmacological insights of this compound. The results confirm that Saq-NO exerted a much stronger protective effect on the in vitro and in vivo cell growth of A375 cells than the parental compound Saq. Saq-NO also inhibited the in vivo growth of A375 cells to a much greater extent than the strong NO-releaser DETA NONOate and its effects were not superimposable to those achieved by the combined treatment of Saq and DETA NONOate. These data demonstrate that Saq-NO represents a NCE with a unique chemotherapeutic profile that can not be reproduced by the combined administration of two related but different molecules, sharing only some features of Saq-NO. The chemicophysical changes conferred by the NO-releasing moiety to the Saq scaffold have clearly induced fundamental changes of the pharmacological actions of the parental Saq molecule such as Akt inhibition. Vice versa, the presence of Saq has plausibly changed the chemico-physical properties of the NO-releasing moiety that might have led to a substantial change in the kinetic profile and/or compartment of NO release resulting in a different pharmacological action.

Both in the in vitro and in vivo studies we have shown that the growth of A375 cells in mice was inhibited by the treatment with either the NO donor DETA NONOate or the NOS inhibitor L-NAME. The effects of the two compounds were of similar magnitude but lower than that achieved with either Saq or Saq-NO.

By using isobologram analysis we have also shown that Saq-NO exerted a synergistic action with the iNOS inhibitor L-NAME and an antagonistic action with the NO donor DETA NONOate on the in vitro growth of A375 cells. These effects were confirmed in nude mice xenotransplanted with A375 cells. On the other hand, Saq did not exert additive or synergistic effects with either L-NAME or DETA NONOate neither in vitro and parallel treatment with Saq and L-NAME just slightly potentiated the reduction of tumor growth in comparison to that observed in monotherapeutic regime and rate of inhibition was less than additive.

The equal efficacy with which the NO donor DETA NONOate and L-NAME prevent the growth of A375 cells in xenografted mice with A375 clearly witnesses how the pleiotropism of NO in the pathogenesis of melanoma may translate into apparently paradoxical therapeutic effects when NO agonists and $\mathrm{NO}$ antagonists are used. This latter observation may mirror the known dichotomic role of NO in the regulation of oncogenesis that may result in pro- or anti-tumor effects depending on concentration, the microenvironment and local or peripheral localization within the tumor. This bimodal action of NO has led this molecule to be considered both as a target for cancer therapy and an anti-cancer agent (19-21). The different contribution of endogenously produced and exogenously-administered NO on the dysregulated growth of A375 cells, and possibly other cancer cells, should also be considered. For example, a study from Chin and Deen (22) anticipates that because of the high rates of cellular consumption, the elevation in NO concentration may be localized, $\sim 90 \%$ of the concentration decay occurring within $30 \mu \mathrm{m}$ of the tumor edge. High concentrations of endogenous NO at the periphery of a melanoma may contribute to metastasis by stimulating cell proliferation, inhibiting apoptosis, or acting as a lymphangiogenic factor. 
In contrast, exogenously-administered NO may also act at more distant sites of the tumor microenvironment and this may results in the activation of anti-oncogenic pathways inhibiting tumor formation. Antitumor action of NO is also manifested through sensitization to apoptosis triggered by mediators of natural immune response. This is very important especially in the light of the fact that most of diagnosed malignancies are already resistant to death signals delivered from surrounding tissues and immune cells. In addition, the different NO concentrations ensuing from low physiological amounts of endogenously produced NO or larger amounts of $\mathrm{NO}$ released from NO donors may generate different concentrations of other closely related reactive nitrogen species (RNS) such as $\mathrm{ONOO}^{-}$. Different $\mathrm{NO}$ concentrations, and/or different RNS, on different cell/tissue compartments can reach totally different molecular targets triggering completely different, if not opposite, pharmacological reactions. This may, for example, explain the dichotomic effects DETA NONOate in the in vitro vs. the in vivo setting. Whilst DETA NONOate treatment was presently effective in reducing A375 growth in vivo, Yang et al have demonstrated that it may promote melanoma progression in vitro by induction of Apurinic/ Apyrimidinic Endonuclease endonuclease-1/redox factor-1 (APE/Ref-1) and related downstream targets such as activator protein-1/JunD, matrix metalloproteinase-1, Bcl-2, and iNOS (17). Similar paradoxes have been also observed in other cancer diseases. For example, despite strong correlation between eNOS expression/activity and poor prostate cancer prognosis (23), the moderate multi-functional NO-donor such as Saq-NO effectively inhibited the in vitro and in vivo growth of human hormone refractory prostate cancer (12). In the same manner, whilst increased NOS2 predicts poor survival in estrogen receptor-negative breast cancer patients (24) and has been thought to play a major pathogenetic role in disease development (25), McMurtry et al (26) have demonstrated that JS-K, a NO-releasing prodrug, induces breast cancer cell death while sparing normal mammary epithelial cells.

The presently observed inhibitory activity of L-NAME in the growth of melanoma is consistent with recent data from Sikora et al (27) who found that oral treatment with the specific iNOS-selective small molecule antagonist N(6)-(1-iminoethyl)l-lysine-dihydrochloride (L-nil) significantly inhibited the growth of two human melanoma cell lines mel624 and mel528 xenotransplanted into severe combined immunodeficient (SCID) mice and extended the survival of tumor-bearing animals. In their experiments, iNOS inhibition was associated with tumor growth suppression, decrease in tumor microvessels, down-regulation of the anti-apoptotic gene Bcl-2, increased number of intratumoral apoptotic cells and enhanced efficacy when L-nil treatment was combined with cisplatin in vivo. This synergism closely resembles that observed in our study between L-NAME and both Saq and Saq-NO. Although dismantling the molecular and pharmacological mechanisms underlying this synergism was outside the phenomenological scope of our work and has not been studied, it is possible that in a similar manner to that described by Sikora et al (27) for N(6)(1-iminoethyl)-1-lysine-dihydrochloride (L-nil) and cisplatin it may be due to combined and higher effective influence on key oncogenic pathways of A375 cells. Taken together these data support the hypothesis that targeting NOS may represent a valuable novel target of melanoma therapies that may be combined with other chemotherapeutic agents including soft NO donor such as Saq-NO.

\section{Acknowledgements}

This work was partly supported by the Ministry of Education and Science of the Republic of Serbia, grant no. 173013.

\section{References}

1. Parkin DM, Bray F, Ferlay J and Pisani P: Global cancer statistics, 2002. CA Cancer J Clin 55: 74-108, 2005.

2. Jemal A, Siegel R, Ward E, Hao Y, Xu J and Thun MJ: Cancer statistics, 2009. CA Cancer J Clin 59: 225-249, 2009.

3. Hodi FS, O'Day SJ, McDermott DF, et al: Improved survival with ipilimumab in patients with metastatic melanoma. N Engl J Med 363: 711-723, 2010.

4. Bedikian AY, Johnson MM, Warneke CL, et al: Does complete response to systemic therapy in patients with stage IV melanoma translate into long-term survival? Melanoma Res 21: 84-90, 2011.

5. Petrella T, Quirt I, Verma S, Haynes AE, Charette M and Bak K; Members of the Melanoma Disease Site Group of Cancer Care Ontario's Program in Evidence-Based Care: Single-agent interleukin-2 in the treatment of metastatic melanoma. Curr Oncol 14: 21-26, 2007.

6. Garbe C,EigentlerTK, Keilholz U,Hauschild A and Kirkwood JM: Systematic review of medical treatment in melanoma: current status and future prospects. Oncologist 16: 5-24, 2011

7. Andersen MH, Junker N, Ellebaek E, Svane IM and Thor Straten P. Therapeutic cancer vaccines in combination with conventional therapy. J Biomed Biotechnol 2010: 237623, 2010.

8. Hershkovitz L, Schachter J, Treves AJ and Besser MJ: Focus on adoptive $\mathrm{T}$ cell transfer trials in melanoma. Clin Dev Immunol 2010: 260267, 2010.

9. Maksimovic-Ivanic D, Mijatovic S, Miljkovic D, et al: The antitumor properties of a nontoxic, nitric oxide-modified version of saquinavir are independent of Akt. Mol Cancer Ther 8: 1169-1178, 2009

10. Mijatovic S, Maksimovic-Ivanic D, Mojic M, et al: Cytotoxic and immune-sensitizing properties of nitric oxide-modified saquinavir in iNOS-positive human melanoma cells. J Cell Physiol 226: 1803-1812, 2010.

11. Rothweiler F, Michaelis M, Brauer P, et al: Anticancer effects of the nitric oxide-modified saquinavir derivative saquinavirNO against multidrug-resistant cancer cells. Neoplasia 12: 1023-1030, 2010.

12. Donia M, Maksimovic-Ivanic D, Mijatovic S, Mojic M, Miljkovic D, Timotijevic G, Fagone P, Caponnetto S, Al-Abed Y, McCubrey J, Stosic-Grujicic S and Nicoletti F: In vitro and in vivo anticancer action of Saquinavir-NO, a novel nitric oxidederivative of the protease inhibitor saquinavir, on hormone resistant prostate cancer cells. Cell Cycle 10: 492-499, 2011.

13. Tang $\mathrm{CH}$ and Grimm EA: Depletion of endogenous nitric oxide enhances cisplatin-induced apoptosis in a p53-dependent manner in melanoma cell lines. J Biol Chem 279: 288-298, 2004.

14. Ekmekcioglu S, Ellerhorst J, Smid CM, et al: Inducible nitric oxide synthase and nitrotyrosine in human metastatic melanoma tumors correlate with poor survival. Clin Cancer Res 6: 4768-4775, 2000.

15. Ekmekcioglu S, Ellerhorst JA and Prieto VG: Tumor iNOS predicts poor survival for stage III melanoma patients. Int J Cancer 119: 861-866, 2006.

16. Johansson CC, Egyházi S, Masucci G, et al: Prognostic significance of tumor iNOS and COX-2 in stage III malignant cutaneous melanoma. Cancer Immunol Immunother 58: 1085-1094, 2009.

17. Yang Z, Yang S, Misner BJ, Chiu R, Liu F and Meyskens FL Jr: Nitric oxide initiates progression of human melanoma via a feedback loop mediated by apurinic/apyrimidinic endonuclease-1/ redox factor- 1 , which is inhibited by resveratrol. Mol Cancer Ther 7: 3751-3760, 2008.

18. White N, Knight GE, Butler PE and Burnstock G: An in vivo model of melanoma: treatment with ATP. Purinergic Signal 5: 327-333, 2009.

19. Huerta S, Chilka S and Bonavida B: Nitric oxide donors: novel cancer therapeutics (Review). Int J Oncol 33: 909-927, 2008. 
20. Hickok JR and Thomas DD: Nitric oxide and cancer therapy: the emperor has NO clothes. Curr Pharm Des 16: 381-391, 2010.

21. Hirst D and Robson T: Nitric oxide in cancer therapeutics: interaction with cytotoxic chemotherapy. Curr Pharm Des 16: 411-420, 2010.

22. Chin MP and Deen WM: Prediction of nitric oxide concentrations in melanomas. Nitric Oxide 23: 319-326, 2010.

23. Nanni S, Benvenuti V, Grasselli A, et al: Endothelial NOS, estrogen receptor beta, and HIFs cooperate in the activation of a prognostic transcriptional pattern in aggressive human prostate cancer. J Clin Invest 119: 1093-1108, 2009.

24. Glynn SA, Boersma BJ, Dorsey TH, et al: Increased NOS2 predicts poor survival in estrogen receptor-negative breast cancer patients. J Clin Invest 120: 3843-3854, 2010.
25. Ambs S and Glynn SA: Candidate pathways linking inducible nitric oxide synthase to a basal-like transcription pattern and tumor progression in human breast cancer. Cell Cycle 10: 619-624, 2011.

26. McMurtry V, Saavedra JE, Nieves-Alicea R, Simeone AM, Keefer LK and Tari AM: JS-K, a nitric oxide-releasing prodrug, induces breast cancer cell death while sparing normal mammary epithelial cells. Int J Oncol 38: 963-971, 2011.

27. Sikora AG, Gelbard A, Davies MA, et al: Targeted inhibition of inducible nitric oxide synthase inhibits growth of human melanoma in vivo and synergizes with chemotherapy. Clin Cancer Res 16: 1834-1844, 2010. 